\title{
Interoperability of Wireless Networks with 4G Based on Layer Modification
}

\author{
Dilshad Mahjabeen ${ }^{1}$, Aminul Haque Mohammed Sayem ${ }^{1}$, Anis Ahmed ${ }^{2}$, Shahida Rafique ${ }^{2}$ \\ ${ }^{1}$ Electrical and Electronics Engineering, Stamford University Bangladesh, Dhaka, Bangladesh \\ ${ }^{2}$ Applied Physics, Electronics and Communication Engineering, University of Dhaka, Dhaka, Bangladesh \\ E-mail:dilshadman@yahoo.com \\ Received February 26, 2010; revised March 21, 2010; accepted April 23, 2010
}

\begin{abstract}
Fourth generation wireless communication systems feel the necessity of transparent and seamless user roaming with end-to-end connectivity. These systems also demand higher data rate, higher mobility support and QoS guarantees due to rapid development of wireless and mobile networks. These requirements open potentials for the operators to increase their service portfolio and for the users to experience context-rich and personalized services. Consequently the interoperability between different wireless network platforms emerges as a crucial necessity. Here focus is given to the significance of the network interoperability aspect based on layered approach and its role in the development towards 4G. This paper also gives an overview of the major $4 \mathrm{G}$ features and differentiating characteristics from other generations.
\end{abstract}

Keywords: 4G, Interoperability, Reconfigurability, Cooperativeness, Cross Layer

\section{Introduction}

Different generations of wireless communication improve the facilities for users day by day. The generations are classified into three groups namely $1 \mathrm{G}, 2 \mathrm{G}$ and $3 \mathrm{G} .1 \mathrm{G}$ was completely analog and used for only voice transmission [1]. $2 \mathrm{G}$ networks were built mainly for voice services and slow data transmission. The cellular services combined with GPRS became $2.5 \mathrm{G}$. This generation provides services such as Wireless Application Protocol (WAP) access, Multimedia Messaging Service (MMS) and for Internet communication services such as email and World Wide Web access. Although $2 \mathrm{G}$ is very popular and successful but it lacks single worldwide radio band technology standard as well as inefficient use of spectrum resources for bursty data. $3 \mathrm{G}$ networks represent the natural evolution from previous standards. In this case the networks enable network operators to offer users a wider range of more advanced services while achieving greater network capacity through improved spectral efficiency. $3 \mathrm{G}$ networks offer a greater degree of security than $2 \mathrm{G}$ predecessors. This generation $(3 \mathrm{G})$ allows simultaneous use of speech and data services and higher data rates. The services provided by $3 \mathrm{G}$ are wide-area wireless voice telephone, video calls, and wireless data, all in a mobile environment [2]. However $3 \mathrm{G}$ performances may not be sufficient to meet needs of future high quality applications. 3G does not provide mobility and service portability since it is based on primarily a wide-area concept. For this facility hybrid network (wireless LAN concept and cell or base-station wide area network design) is required. We need all digital packet networks that utilize IP in its fullest form with converged voice and data capability. And this great opportunity will be fulfilled by $4 \mathrm{G}$ wireless communications. In this paper we mainly deal with interoperability - the most important and special characteristic of $4 \mathrm{G}$ wireless communications. Section II describes the characteristics of $4 \mathrm{G}$ and the motivation for $4 \mathrm{G}$ other than $3 \mathrm{G}$. This section also differentiates $4 \mathrm{G}$ from other generations. Section III focuses how $4 \mathrm{G}$ based on layered modification provide interoperability. Section IV describes about the role of cross layer at interoperable issue by $4 \mathrm{G}$.

\section{Journey towards 4G from 3G}

The limitations of previous generation lead to improved generation. Their characteristics vary depending on some demanding features. Some of the differentiating characteristics are given in Table 1. The first generation of cellular networks consisted of analog systems capable of carrying only voice. $2 \mathrm{G}$ were packet switched transferring voice only. $3 \mathrm{G}$ is basically a circuit switched cellu- 
lar network and so they have their own gateway to interpret IP from the back bone network. They also have their own protocol and interfaces for communication within themselves. To make this problem end, the only solution is $4 \mathrm{G}$ networks. Moreover, $3 \mathrm{G}$ is lacking from the followings: limitation of spectrum allocation, challenging gradually increasing bandwidth and high data rate for multimedia service, difficulty to roam across distinct service environment, lack of end environment, and lack of end to end continuous transmission mechanism [3]. To face these challenges, the new level of mechanism, $4 \mathrm{G}$ communications is introduced.

4G is an all IP packet switched network. This generation is the upgrade strategy in world of wireless communications. 4G system is expected to provide a comprehensive and secure IP based solution with facilities like voice, data and streamed multimedia. The key characteristics of $4 \mathrm{G}$ are global mobility, service portability, scalability and seamless handoff. It will be very friendly to comprehensive like "Anytime, Anywhere, Anyhow and Always-on" basis and at much higher data rates compa- red to previous generations. This will secure IP based solution with facilities like voice, data and streamed multimedia. Another special characteristic of $4 \mathrm{G}$ is the interoperability with existing wireless standards [4]. This generation provides integration across different network topologies i.e., hybrid network architecture that integrates wireless wide area networks wireless.

\section{Interoperability}

With the rapid development of various wireless communication systems worldwide, there are also gradual changes in users' expectation and demand. Consequently the corresponding wireless networks work many fold at their capacity limits. So there is every chance of emergency crisis and/or disasters at the peak and crucial period. Thus interoperability can offer network providers with a possibility to switch between alternative wireless access networks. The basic theme of interoperability will yield the necessity of (user transparent) reconfigurability and

Table 1. Comparison of different generations.

\begin{tabular}{|c|c|c|c|}
\hline Properties & $2 \mathrm{G}$ & $3 \mathrm{G}$ & $4 \mathrm{G}$ \\
\hline Network Architecture & LAN, & Wide area cell-based & Hybrid - \\
\hline Driving Architecture & Only voice & dominantly voice; also data & Converged data and voice over IP \\
\hline Switching & Packet switched & Circuit and Packet & All digital with packetized voice \\
\hline Radio Access & FDMA, TDMA, CDMA & WCDMA, CDMA2000, IWC-136 & MC-CDMA, OFDMA \\
\hline Database & HLR,VLR, EIR, AuC & EHLR, VLR, EIR, AuC & EHLR, VLR, EIR, AuC \\
\hline Data rates & 9.6 to $384 \mathrm{kbps}$ & Up to $2 \mathrm{Mbps}$ & 100Mbps \\
\hline Roaming & Restricted & Global & Global \\
\hline Compatible & Not compitable to $3 \mathrm{G}$ & $\begin{array}{l}\text { Compitable to } 2 \mathrm{G}, 2 \mathrm{G}+\text { and blu- } \\
\text { tooth }\end{array}$ & Compatible to $3 \mathrm{G}$ \\
\hline Handsets & $\begin{array}{l}\text { Dual mode TDMA and CDMA } \\
\text { Voice and data terminals }\end{array}$ & $\begin{array}{l}\text { Multiple mode voice, data, vedio } \\
\text { terminals }\end{array}$ & $\begin{array}{l}\text { Multiple mode voice, data streamed } \\
\text { video at higher data rates. }\end{array}$ \\
\hline $\begin{array}{l}\text { Applica- } \\
\text { tions }\end{array}$ & SMS, Internet & Internet, SMS & $\begin{array}{l}\text { Internet, MMS, Mutimedia, } \\
\text { HDTV, M TV }\end{array}$ \\
\hline Bandwidth & $25 \mathrm{MHz}$ & $5-20 \mathrm{MHz}$ & $100 \mathrm{MHz}$ \\
\hline Frequency Band & Tri Band800, 900,1800,1900 MHz & $\begin{array}{l}\text { Dependent on country }(1800-2400 \\
\mathrm{MHz})\end{array}$ & Higher frequency bands $(2-8 \mathrm{GHz})$ \\
\hline Component design & Optimized antenna design & $\begin{array}{l}\text { Optimized antenna design, } \\
\text { multi-band adapters }\end{array}$ & $\begin{array}{l}\text { Smarter Antennas, software multiband } \\
\text { and wideband radios }\end{array}$ \\
\hline IP & $\begin{array}{l}\text { No IP } \\
\text { Connection }\end{array}$ & $\begin{array}{l}\text { A number of air link protocols, } \\
\text { including IP } 5.0\end{array}$ & All IP (IP6.0) \\
\hline
\end{tabular}


cooperativeness in various communication systems tunneling towards the $4 \mathrm{G}$ journeys.

\subsection{Reconfigurability}

The reconfigurable interoperability can be done at the network level, the user level or both. This will be very helpful for both the network providers and the users' perspective. The reconfigurable interoperability will provide selection between alternative wireless access networks. The selection could be based on several reconfigurable interoperability issues such as:

- Channel state;

- $\quad$ Outage probability;

- Vertical handover probability;

- Users' QoS requirements;

- Context awareness;

- $\quad$ Load sharing and distribution between different spatially coexisting wireless networks;

- $\quad$ Efficient spectrum sharing;

- $\quad$ Preferred gateway selection and network discovery and

- Congestion control.

The mechanism of reconfigurability refers not only to the physical layer, but span across the entire protocol stack (including cross-layer optimizations). The reconfigurable interoperability of the heterogeneous $4 \mathrm{G}$ system will lead to more efficient end-to-end connectivity and service delivery in heterogeneous environments, easier worldwide roaming and dynamic adaptation to regional contexts, enhanced personalization and richer services. At the network level, the reconfigurable interoperability will offer network providers with a possibility to choose between alternative wireless accesses networks at minimal cost. At the user level, the interoperability of the heterogeneous $4 \mathrm{G}$ systems will provide more efficient end-to-end connectivity and service delivery in heterogeneous environments, easier global roaming and dynamic adaptation to regional contexts, enhanced personalization and enriched services [5].

\subsection{Cooperativeness}

3G specify the PHY and MAC of the radio link. This alone is not adequate to build an interoperable broadband wireless network. Interoperable networks involve the following issues:

- $\quad$ End-to-end service such as IP connectivity;

- Session management;

- Security;

- QoS;

- Mobility;

- Connectivity issues;

- Self organization;
- Authentication, Authorization, and Accounting.

Cooperativeness comes to ensure these issues. This means connectivity between all the entities of a network in a consistent manner across all access technologies for any service. In $4 \mathrm{G}$, a cooperative network (CoNet) consists of three distinct layers such as application, connectivity and access that form logically separate subsystems. Each of the layers can be further divided into different sub layers as shown in the Figure 1. The layers should have well defined interfaces and be functionally independent of each other for an approach is required to ensure easy adaptation of heterogeneous access technologies, related technology changes, and flexible support for rapid service innovation. Actually the connectivity layer plays an important role for cooperation across various realizations of networks, which in turn ensures the interoperability. This layer will be independent of the various transport technologies used to link the nodes of the network together. Finally, the user will enjoy seamless roaming across different access technologies and administrative domains without any manual user intervention [6].

One of the 4G's major goals is integration, which offers seamless interoperability of different types of wireless networks with the wire line backbone. Some of the available attempted heterogeneous interoperable integrated architecture are: a loosely-coupled, Mobile IPv6 (MIPv6)-based GPRS/WLAN/LAN heterogeneous network, implementation of IPv6-based mobility-enabled network architecture with Authentication, Authorization, Accounting and Charging (AAAC) services and support for Quality of Service (QoS) [7].

\subsection{Access Network}

From the point of view of access network, 3G access

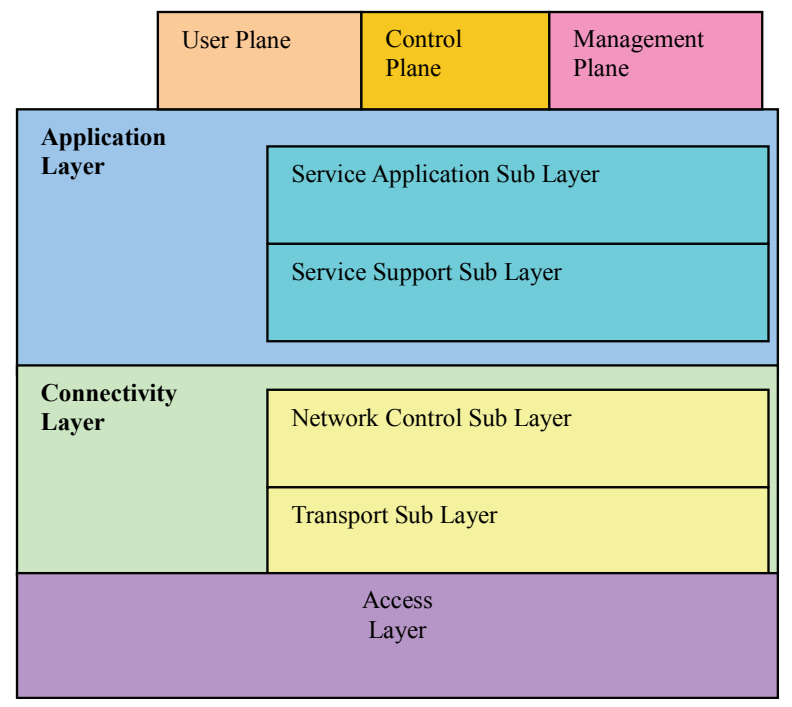

Figure 1. Layers of $\mathbf{4 G}$ providing interoperability. 
network uses WCDMA, cdma 2000. But these are complicated and require more protocol for system structure coverage. On the other hand, $4 \mathrm{G}$ access network uses the OFDMA, $3 \times$ RTT and MIMO antennas. Also hybrid multiple access technique is used for high speed mobile or nomadic user, data or voice traffic, call centre or boundary conditions [8]. But the abovementioned access techniques currently do not interoperate LAS CDMA (Large area synchronized CDMA) access technique solves this problem. LAS CDMA will be compatible with all current and future standards and there is a relatively easy transition from the existing system to LAS CDMA. Link air emphasizes that LAS CDMA will accommodate all the advanced technology planned for 4G. LAS CDMA will also further improve the prevailing the techniques like WCDMA, $3 \times$ RTT [1].

\section{Cross Layer}

In wireless network, interoperable systems provide coordination among layers. Cross-layer design or "cross-layering" provides functionalities associated with the original layers to allow coordination, interaction and joint optimization of protocols crossing different layers. In order to provide improvement in terms of some performance metric, the cross-layer approach to system design derives from the interaction among protocols operating at different layers of the protocol stack. The main advantage deriving cross layering paradigm is the modularity in protocol design, which enables interoperability and improved design of communication protocols. An example of cross layer approach for interoperability is shown in Figure 2.

MAC-PHY Cross Layer: The physical layer transmits power, which can be tuned by the Medium Access Control (MAC) layer to increase the range of transmission.

NET-MAC Cross Layer: Network layer could use

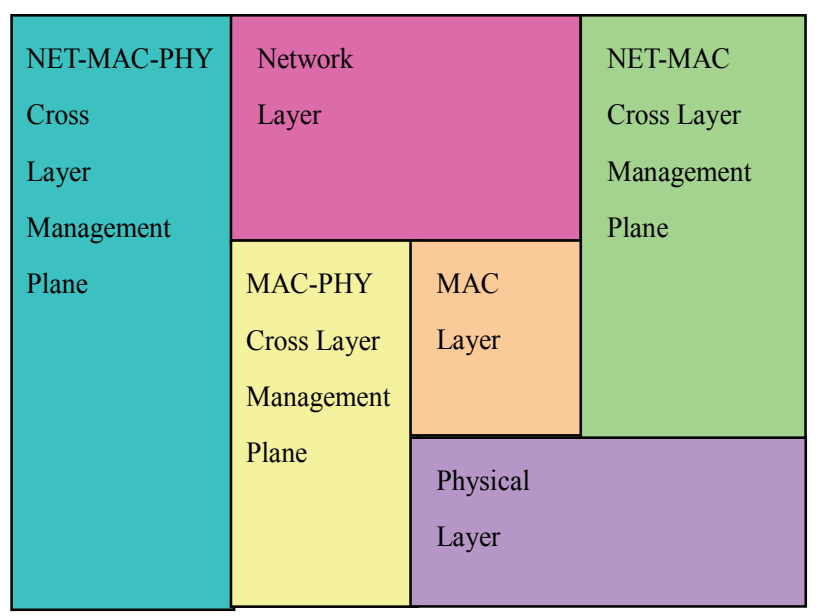

Figure 2. Cross-Layer approach for Interoperability.
MAC layer events like handoff to reduce Mobile-IP hand-off latency for seamless connectivity.

NET-MAC-PHY Cross Layer: This layer provides seamless connectivity and enhanced transmission range [9].

\section{Conclusions}

For higher data rates, higher mobility support and seamless communication $4 \mathrm{G}$ utilizes a common platform that will unify a variety of evolving access technologies, uninterupted internetworking and interoperability solutions and adaptive multimode user terminals. Reconfigurable, co-operative and cross layer architecture based on layered approach for interoperability are mentioned here. The co-net architecture also provides end to end services, security and self organization. More over using multiple descriptions coding at application layer combined with orthogonal frequency division multiplexing at the link level provides robustness against hostile wireless channels. Negotiation between application, data link control and physical layer is exploited to increase user quality of service in terms of picture signal to noise ratio and bandwidth efficiency. 4G networks suffer from the lack of Layer 2 QoS provisioning in heterogeneous networks, mainly due to the non-uniform nature of the QoS models and service interfaces among different wireless technologies. Other problem is the lack of coordination of L3 QoS with L2 QoS and mobility. All these problems can be solved by introducing QoS abstraction layer in between layer 2 and 3 in the control plane which will be discussed in our next paper.

\section{References}

[1] "Emerging Wireless Technologies: A Look into the Future of Wireless Communications-Beyond 3G". http://www.safecomprogram.gov/NR/rdonlyres/5C74C631ACF6-433F-B313-C

[2] D. I. Axiotis, F. I. Lazarakis and C. Vlahodimitro, "Mobility and Traffic Parameters for Simulating Interoperating UMTS and HIPERLAN/2 MTMR Enabled Networks," The 57th IEEE Seminual Vehicular Technological Conference, Jeju,Vol. 4, 2003, pp. 2745-2749.

[3] S. Hussain, Z. Hamid and N. S. Khattak, "Mobility Management Challenges and Issues in 4G Heterogenous Networks," ACM, New York, USA, 2006.

[4] "Evolution of wireless connection". http://4G-wirelessevolution.tmcnet.com/topicsmostigov/ $\mathrm{my} / \mathrm{mosti} /$ images/stories/DICT/policy

[5] L. M. Gavrilovska and V. M. Atanasovski, "Interoperability in Future Wireles Communications Sytems: A Roadmap to 4G," Microwave Review,Vol. 13, No. 1, 2007, pp. 19-28. 
[6] "Cooperative Networks of 4G". www-scf.usc/edu/ ssaraf/ EE555.pdf

[7] L. Gavrilovska, V. Atanasovski, V. Rakovic, O. Ognenoski and A. Momiroski, "Providing Interoperability in Heterogeneous Environments towards 4G," ELMAR, 50th International Symposium, Zadar, Vol. 1, 2008, pp. 223-226.

[8] L. D. Uomo and E. Scarrone, "All-IP 4G Network architecture for Efficient Mobility and Resource Management," 5th International Symposium on Wireless Personal
Multimedia Communications, Honolulu, Vol. 2, 2002, pp. 591-595.

[9] D. Kliazovich, M. Devetsikiotis and F. Granelli, "Formal Methods in Cross Layer Modeling and Optimization of Wireless Networks," In: Kotsopoulos, S. and Ioannou, K. Ed., Handbook of Research on Heterogeneous next Generation Networking: Innovations and Platforms, 2009, pp. 1-24. 